\title{
Extracellular signals and receptor-like kinases regulating ROP GTPases in plants
}

\author{
Kaori N. Miyawaki ${ }^{1}$ and Zhenbiao Yang ${ }^{2 *}$ \\ 'Shanghai Center for Plant Stress Biology, Shanghai, China \\ ${ }^{2}$ Center for Plant Cell Biology, Department of Botany and Plant Sciences, University of California, Riverside, CA, USA
}

\section{Edited by:}

Shin-ichi Arimura, The University of

Tokyo, Japan

Reviewed by:

Frantisek Baluska, University of Bonn, Germany

Michael Sauer, Centro Nacional de Biotecnología - Consejo Superior de Investigaciones Científicas, Spain

\section{${ }^{*}$ Correspondence:}

Zhenbiao Yang, Center for Plant Cell Biology, Department of Botany and Plant Sciences, University of California, 900 University Avenue, Riverside, CA 92521, USA e-mail:yang@ucr.edu
Rho-like GTPase from plants (ROPs) function as signaling switches that control a wide variety of cellular functions and behaviors including cell morphogenesis, cell division and cell differentiation. The Arabidopsis thaliana genome encodes 11 ROPs that form a distinct single subfamily contrarily to animal or fungal counterparts where multiple subfamilies of Rho GTPases exist. Since Rho proteins bind to their downstream effector proteins only in their GTP-bound "active" state, the activation of ROPs by upstream factor(s) is a critical step in the regulation of ROP signaling. Therefore, it is critical to examine the input signals that lead to the activation of ROPs. Recent findings showed that the plant hormone auxin is an important signal for the activation of ROPs during pavement cell morphogenesis as well as for other developmental processes. In contrast to auxin, another plant hormone, abscisic acid, negatively regulates ROP signaling. Calcium is another emerging signal in the regulation of ROP signaling. Several lines of evidence indicate that plasma membrane localized-receptor like kinases play a critical role in the transmission of the extracellular signals to intracellular ROP signaling pathways. This review focuses on how these signals impinge upon various direct regulators of ROPs to modulate various plant processes.

Keywords: ROP GTPase, RLK, auxin, abscisic acid (ABA), calcium

\section{INTRODUCTION}

The Rho family of small GTPases is conserved and plays pivotal roles in cellular signaling in all eukaryotic kingdoms. In animals and fungi, the Rho family is divided into Rac, Rho, and CDC42 subfamilies, each with unique functions. Plants contain a single subfamily, named Rho-like GTPase from plants (ROPs) which appears to have evolved prior to the divergence of fungal and animal Rac, Rho, and CDC42. The Arabidopsis thaliana genome encodes 11 ROPs and most of them are associated with the plasma membrane (PM), where they transmit the signal from membranelocalized receptors (Li et al., 2001; Fu et al., 2002; Gu et al., 2004; Nibau et al., 2006; Yang, 2008; Craddock et al., 2012). Thus the regulation of their membrane association is important for ROP signaling. ROP signaling can be negatively regulated by guanine nucleotide dissociation inhibitors (GDIs), which are responsible for the dissociation of ROPs from the PM and by sequestering them in the cytosol in inactive GDP-bound forms. When associated with the PM, ROP proteins shuttle between inactive GDP-bound form and active GTP-bound form. They bind to their downstream effector proteins only when they are in the GTP-bound active status. Once the upstream signals are perceived by receptors, guanine nucleotide exchange factors (GEFs) replace the GDP bound to ROPs with GTP. RopGEFs have a conserved plant specific ROP nucleotide exchanger (PRONE) domain for GEF activity. In contrast to RopGEFs, GTPase-activating proteins (GAPs) promote GTP hydrolysis of ROP proteins. The activation of ROPs by upstream factor(s) is a critical step in the regulation of ROP signaling. Several lines of evidence show that ROPs have roles in signaling pathway mediated by some plant hormones, such as auxin and abscisic acid (ABA). Recent findings showed that auxin is an important signal for the activation of ROPs during leaf epidermal pavement cell (PC) morphogenesis as well as in other developmental processes such as root hair development. In both cases, PM localized-receptor like kinases (RLKs) play critical roles for transmitting an extracellular auxin signal to intracellular ROP signaling. By contrast, ROPs are inactivated by ABA. In addition to plant hormones, calcium is an emerging signal in the regulation of ROP signaling in pollen tube growth. This review focuses on the mechanism underlying upstream regulation of ROP signaling and on how these signals impinge upon various direct regulators of ROPs to modulate various plant developmental processes.

\section{EXTRACELLULAR AUXIN SIGNAL ACTIVATES ROP SIGNALING}

Leaf epidermal PC is a well-established model system for the study of ROP signaling in Arabidopsis (Fu et al., 2002, 2005; Yang, 2008; Figures $\mathbf{1}$ and $\mathbf{2 A}$ ). These cells form a puzzle piece shape with interdigitated lobes and indentations, and their developmental processes are involved in the establishment of multi-polarity within each cell and the coordination of the multi-polarity between adjacent cells. ROP signaling plays a critical role in regulating the formation of both lobes and indentations during PC patterning. ROP2 and ROP4, two functionally overlapping ROPs, promote the lobe growth by activating the ROP-interactive CRIB motif containing protein 4 (RIC4)-mediated accumulation of fine cortical actin microfilaments (MFs). On the other hand, ROP2 and ROP4 inactivate ROP-interactive CRIB motif containing protein 1 (RIC1)-mediated microtubule (MT) bundling by 


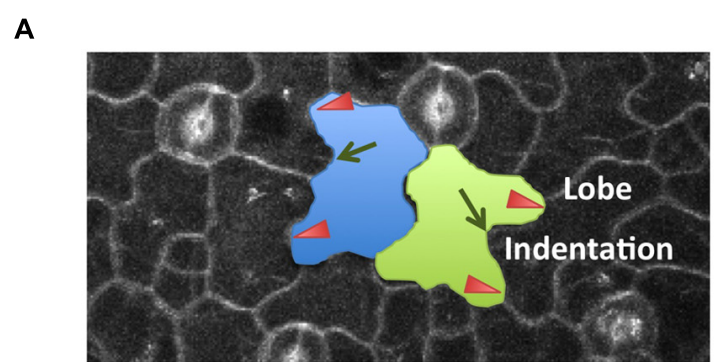

B

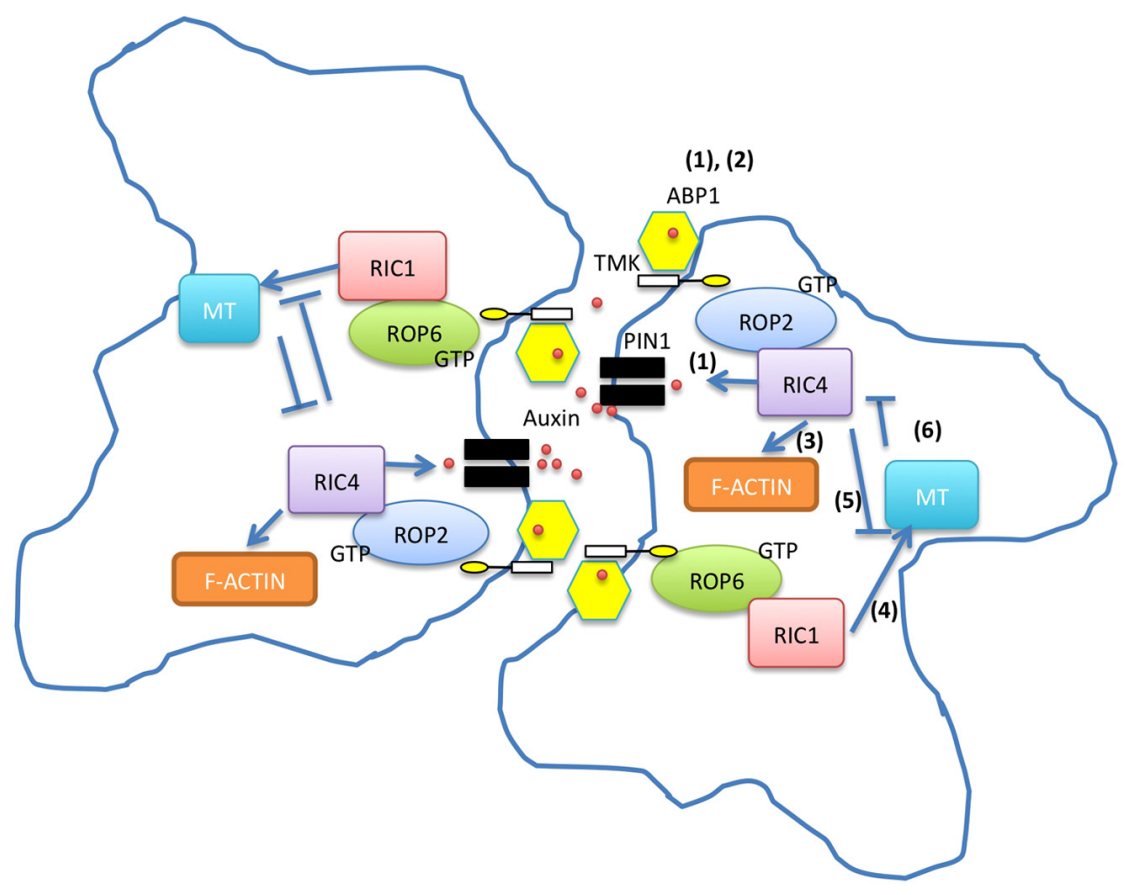

FIGURE 1 | Two antagonistic Rho-like GTPase from plant (ROP) pathways regulate pavement cell (PC) interdigitation. (A) Lobes and indentations in Arabidopsis PC. Red arrowheads indicate lobes. Green arrows show indentations. (B) Auxin activates two antagonistic ROP pathways in PC interdigitation (1) Apoplastic auxin localizes to the lobes by PIN1-mediated positive feedback loop. Accumulation of extracellular auxin is generated by the auxin->ROP2->PIN1->auxin feedback loop. (2) Auxin controls ROP2 and ROP6 pathways in an ABP1/TMK-dependent manner. (3) ROP2 activates RIC4 and promotes assembly of fine cortical actin microfilaments in lobe regions. (4) ROP6 activates ROP-interactive CRIB motif containing protein 1 (RIC1) and promotes cortical microtubule (MT) ordering in indenting regions. (5) ROP2-mediated pathway inactivates RIC1-mediated MT bundling. (6) RIC1-mediated MT organization inhibits ROP2/RIC4 interaction. disrupting its RIC1-association with cortical MTs (Fu et al., 2002, 2005). In contrast, ROP6 promotes cortical MT ordering through RIC1 to restrict radial cell expansion in indenting regions. Both RIC1 overexpression and cortical MT polymerization inhibited ROP2-RIC4 interaction, indicating that RIC1-mediated MT organization antagonized the ROP2/RIC4 pathway (Fu et al., 2005, 2009).

Auxin promotes the interdigitation of PCs in Arabidopsis (Xu et al., 2010). Xu et al. (2010) demonstrated that abnormal PC phenotypes of mutants lacking four auxin biosynthetic enzyme genes, YUCCA, were suppressed by auxin treatment. They further showed that two antagonistic ROPs, ROP2, and ROP6, are activated in an auxin-dependent manner. The application of exogenous auxin rapidly activates both ROP2 and ROP6 within minutes, suggesting that auxin activates PC interdigitation independent of the well-studied auxin receptors, TRANSPORT INHIBITOR RESPONSE1/AUXIN SIGNALING F-BOX (TIR1/AFBs), which regulate auxin-mediated gene expression in the nucleus. Instead, it was shown that activation of ROP2 and ROP6 by auxin requires AUXIN BINDING PROTEIN1 (ABP1). ABP1 is a 22-kDa glycoprotein, carrying the endoplasmic reticulum (ER) retention signal KDEL sequence and was implicated as an extracellular auxin receptor for the regulation of rapid auxin responses such as auxin promotion of cell expansion (Napier et al., 2002; Braun et al., 2008; Tromas et al., 2009; Robert et al., 2010). A portion of ABP1 is localized to the cell surface, although the majority of it remains in ER (Jones and Herman, 1993; Diekmann et al., 1995; Henderson et al., 1997; Napier et al., 2002). The cell surface action of ABP1 is consistent with a role for ABP1 in the activation of ROPs in PCs. The defects in PC interdigitation in either the $a b p 1-5$, containing a 




point mutation (His94->Tyr) in the auxin binding-pocket, or an inducible $A B P 1$ antisense line were not rescued by auxin, suggesting that ROP2 and ROP6 signaling is regulated downstream of ABP1 (Xu et al., 2010). ROP2 is required for the targeting of the auxin efflux transporter, PINFORMED 1 (PIN1), to the lobing regions by inhibiting PIN1 endocytosis, suggesting that the local high levels of auxin, induced by auxin efflux, contribute to the promotion of lobe growth (Xu et al., 2010; Nagawa et al., 2012).

Although ABP1 lacks a transmembrane domain, the requirement of $\mathrm{ABP} 1$ for the rapid activation of ROP signaling by auxin suggests that $\mathrm{ABP} 1$ directly regulates ROP signaling at the PM. It was proposed that apoplastic ABP1 must interact with a transmembrane docking protein to transmit auxin signals to the cytoplasm (Shi and Yang, 2011). A possible docking protein candidate is CBP1, a GPI-anchored protein that binds to the C-terminus of $\mathrm{ABP} 1$ and masks the KDEL ER retention signal (Shimomura, 2006). However, CBP1 lacks any motifs to induce downstream signaling, and thus the presence of other proteins that associate with $\mathrm{ABP} 1$ or form a protein complex with ABP1 is expected. Recently, (Xu et al., 2014) demonstrated that the transmembrane kinases (TMKs), which belong to the family of receptor like kinase, interact with $\mathrm{ABP} 1$ on the cell surface in an auxin-dependent manner to regulate ROP signaling. The four members of the TMK subfamily possess common features; an extracellular domain carrying multiple leucine-rich repeats (LRRs), a transmembrane domain, and an intracellular kinase domain. TMKs have critical roles in cell expansion and cell division downstream of auxin (Dai et al., 2013). The quadruple mutant of tmk1234 exhibited embryonic lethality with less severity than those phenotypes in abp1 null mutants.
The defects of PC interdigitation of $t m k 1234$ were not rescued by auxin. Mutations in four TMKs caused a decrease in active ROP2 and ROP6 in the presence of exogenous auxin as well as reduced RIC4 localization at the PM and RIC1 interaction with cortical MTs in PC. These results suggested that TMKs are required for both ROP2 and ROP6 activation in PC patterning. Furthermore, biochemical approaches revealed that the extracellular domain of TMK1 physically interacts with ABP1 in an auxin-dependent manner. Thus, TMK1 is a long-sought docking protein, which is required to transmit the ABP1-mediated auxin signal to the downstream ROP signaling pathways. These findings also confirmed that ABP1 is indeed an extracellular auxin receptor and provide the first example of an extracellular signal that activates ROP signaling at the PM.

The regulation of ROP signaling by extracellular auxin appears to be a common signaling mechanism in plants. Apoplastic ABP1 promotes clathrin-dependent endocytosis, which leads to PIN internalization in roots. Auxin binding to ABP1 inhibits the positive action of ABP1 in endocytosis (Robert et al., 2010; Figure 2B). Genetic analysis further revealed that ROP6/RIC1 act downstream of ABP1 to regulate clathrin-dependent endocytosis (Chen et al., 2012). SPIKE1 (SPK1), a ROP guanine nucleotide exchange factor, is required for ROP6 activation by auxin (Lin et al., 2012; Figure 2B). The SPK1-ROP6-RIC1 signaling pathway was demonstrated to regulate PIN2 distribution through the regulation of clathrin-dependent endocytosis in roots. It will be interesting to determine whether TMKs are also involved in ROP signaling downstream of auxin that inhibits PIN endocytosis in roots.

Auxin can also regulate root hair elongation through ROP signaling. Root hair derives from a single epidermal hair-forming cell, followed by tip-growth at apex of the cell as observed in pollen tube growth. Immunolocalization of the ROP4 protein and the localization of GFP-ROP2 revealed that these ROPs are localized to the tip of elongating root hair cells. The constitutively-active (CA) form of ROP2, ROP4, and ROP6 induced isotropic growth and elongation of root hair cells, whereas the dominant-negative (DN) form of ROP2 inhibited the tip growth of root hair (Molendijk et al., 2001; Jones et al., 2002; Yang, 2002; Duan et al., 2010) identified the receptor-like kinase FERONIA (FER), as an upstream regulator of ROP signaling by using RopGEF1 as the bait in a yeast twohybrid screen. Several lines of evidence suggest that the complex formed by FER and RopGEF1 recruits an inactive form of ROP2 and converts it to an active form. Auxin was reported to stimulate root hair elongation (Pitts et al., 1998; Rahman et al., 2002). Root hairs of fer mutants are insensitive to exogenous application of auxin, whereas GFP-ROP2 overexpression restored root hair elongation of a fer mutant. The fer mutants showed decreased accumulation of active ROPs, and the pull-down assay revealed that the inactive (GDP) form of ROP2 preferentially binds to FER. These data suggest that FER is an upstream receptor of ROP signaling in the auxin-dependent root hair development. FER activates NADPH oxidase-dependent production of reactive oxygen species (ROS), which is a second messenger for polar growth (Duan et al., 2010). It would be interesting to investigate whether ABP1-TMKs signaling is involved in the FER-dependent root hair elongation activated by auxin and if so, what the relationship between TMKs 
and FER is. Recently, Haruta et al. (2014) have reported that a secreted peptide RALF (rapid alkalinization factor) directly interacts with FER to suppress root cell elongation, and RALF acts through FER to inactivate the $\mathrm{PM} \mathrm{H} \mathrm{H}^{+}$-ATPase. Auxin promotes $\mathrm{H}^{+}$extrusion through $\mathrm{H}^{+}$-ATPase that causes the lower apoplastic $\mathrm{pH}$, followed by the extension of cell wall. Another recent report showed that auxin induces phosphorylation of the $\mathrm{H}^{+}$-ATPase as well as hypocotyl elongation in a TIR1/AFB-independent manner (Takahashi et al., 2012). Both auxin-induced cell elongation and $\mathrm{H}^{+}$extrusion depend on $\mathrm{K}^{+}$uptake through inward-rectifying $\mathrm{K}^{+}$channels (Claussen et al., 1997; Philippar et al., 2006). Since it has been reported that the $\mathrm{C}$-terminal region of maize ABP1 modulates $\mathrm{K}^{+}$channels coupling to cytoplasmic $\mathrm{pH}$ (pHi; Thiel et al., 1993), ABP1-TMK signaling may control $\mathrm{pH}$ through $\mathrm{H}^{+}$. ATPase. Thus it will be intriguing to determine whether RALF-FER signaling regulates ROP, and to elucidate the crosstalk between RALF-FER signaling and ABP1-TMK signaling. The molecular mechanism underlying FER-mediated pathway may shed light on how multiple signals are integrated in a specific developmental phase.

\section{ROP SIGNALING IS REGULATED BY RECEPTOR LIKE KINASES IN POLLEN TUBE GROWTH}

Arabidopsis ROP1, a pollen-specific member of ROPs, is the central regulator of pollen tube tip growth. The active ROP1 protein is localized in the apical cap of the PM of pollen tubes as an apical cap, corresponding to the expanding region (Lin et al., 1996; Hwang et al., 2005). Inhibition of ROP1 signaling by DNROP1 or microinjection of anti-ROP1 caused pollen tube growth arrest, whereas overexpression of ROP1 and CA-ROP1 induced depolarized pollen tube growth (Lin and Yang, 1997; Li et al., 1999; Gu etal., 2003). ROP1 activates two counteracting pathways; the RIC4-dependent F-actin assembly pathway controls the vesicle accumulation required for tip growth, whilst the RIC3dependent calcium accumulation leads to F-actin disassembly that promotes exocytosis at the tip. (Gu et al., 2005; Lee et al., 2008). Mathematical models and experimental evidence suggest that the RIC4-F-actin pathway contributes to a positive feedback regulation of ROP1 signaling, whereas the RIC3-calcium pathway has a role in a negative feedback regulation of ROP1 signaling (Yan et al., 2009). REN1 (ROP1 enhancer 1) encodes a Rho GTPaseactivating protein (RhoGAP), which confines ROP1 activity to the tip of pollen tube. REN1 localizes in vesicles that accumulate in the apex of pollen tubes and at the PM of pollen tube tip, where ROP1 is activated. It was reported that ROP1 function is required for both the localization and the function of REN1, indicating that both spatial distribution and function of REN1 are downstream of ROP1 signaling (Hwang et al., 2008). Thus REN1 appears to participate in the negative feedback regulation of ROP1 signaling. It will be interesting to determine whether REN1 function in the negative feedback is linked to calcium signaling.

Another outstanding question about the regulation of ROP signaling involves the nature of the initial signal that activates ROP1 to promote pollen tube growth. It appears that the signal may also be extracellular, as another subfamily of RLK has been implicated in the activation of ROP1 signaling in pollen tubes. The two pollen-specific RLKs, LePRK1 and LePRK2 (for Lycopersicon esculentum pollen receptor kinase 1 and 2) were discovered (Muschietti et al., 1998). LePRK1 and LePRK2 are both localized at the surface of elongating pollen tubes. LePRK1 and LePRK2 are coimmunoprecipitated in pollen that suggesting that these two receptors interact with each other (Wengier et al., 2003). The down-regulation of LePRK2 by antisense of LePRK2 expression caused reduced pollen germination and defects in pollen tube growth (Zhang et al., 2008). A kinase partner protein (LeKPP), the pollen specific homolog of RopGEF from tomato, associates with the cytoplasmic region of LePRK2 in pollen. Pollen specific promoter-driven LeKPP resulted in depolarization of pollen tube with abnormal actin assembly (Kaothien et al., 2005). AtRopGEF12, an Arabidopsis homolog of LeKPP, interacts with an Arabidopsis pollen receptor kinase AtPRK2a. The C-terminally truncated form of AtRopGEF12 disturbed pollen tube morphology, whereas full-length AtRopGEF12 caused slightly wider pollen tubes, indicating that the $\mathrm{C}$ terminus has an inhibitory function for GEF activity. A phospho-mimicking mutation at a conserved serine residue in the C terminus of AtRopGEF12 caused the loss of inhibitory effects. Coexpression of AtRopGEF12 and AtPRK2a resulted in isotropic growth of pollen tubes as seen in CA-ROP overepressing lines. Since coexpression of AtPRK2a and an N-terminally truncated form of AtRopGEF12 did not cause the isotropic pollen tube growth, the $\mathrm{N}$ terminal region of AtRopGEF12 appears to play a positive role for the activation of ROP signaling in the pollen tube growth (Zhang and McCormick, 2007). These data suggest that AtPRK2a binds to the C-terminal region of AtRopGEF12, and the self-inhibitory function of GEF activity is lost by phosphorylation at an invariant serine residue, and then the $\mathrm{N}$ terminal region of AtRopGEF12 activates downstream ROP signaling. AtPRK2a possesses a kinase activity (Chang et al., 2013). A recent report showed that not only the C-terminal region but also the juxtamembrane (JM) domain of AtPRK2a is critical for its interaction with AtRopGEF12 and the subcellular distribution of AtPRK2a at the PM, suggesting that AtPRK2a can interact with AtRopGEF12 without phosphorylation (Zhao et al., 2013).

There are fourteen members of the RopGEFs family in Arabidopsis. Transient expression of five AtRopGEFs causes swollen phenotypes in tobacco pollen, as seen in ROP1- or CA-ROP1overexpressed pollen (Lin and Yang, 1997; Li et al., 1999; Gu et al., 2003). AtRopGEF1 overexpression induced the severe swollen phenotypes in a ROP1-dependent manner. The PRONE domain of AtRopGEF1 exhibited GEF activity on ROP1. These data suggest that RopGEF1 directly activates ROP1 (Gu etal., 2006). Chang etal. (2013) showed that AtPRK2 (also referred to as AtPRK2a) induces ROP1 activity and pull-down assay revealed that AtPRK2 physically interacts with AtRopGEF1 and ROP1. AtPRK2 directly phosphorylates AtRopGEF1 in the C-terminal region, which is critical for AtRopGEF1 function ( $\mathrm{Gu}$ et al., 2006; Chang et al., 2013). Furthermore, CA-AtRopGEF1 (PRONE domain of AtRopGEF1) rescued pollen germination defects in the DN-AtPRK2 (a kinase domain-deleted form of AtPRK2) overexpressing line (Chang et al., 2013). These data suggest that the AtPRK2-AtRopGEF1-ROP1 signaling pathway controls pollen tube growth. 
A fascinating outstanding question remains the nature of the extracellular signal that regulates RLKs in pollen tube growth. LAT52, a cysteine-rich extracellular protein from pollen, interacts with LePRK2 (Muschietti etal., 1994; Tang etal., 2002). LeSHY, a leucine-rich repeat protein from pollen, and LeSTIG1, a small cysteine-rich protein from pistil, can bind the extracellular domains of LePRK1 and LePRK2, and exogenous application of LeSTIG1 promotes pollen tube growth (Tang et al., 2004; Huang et al., 2014). It remains unknown whether these signals regulate ROP signaling in tomato. It may be intriguing to identify the signal that binds to AtPRK2 and to examine whether the signal regulates AtPRK2-AtRopGEF1/AtRopGEF12-ROP1 pathway in Arabidopsis pollen tube growth.

\section{PAN1 RLK AND ROP SIGNALING REGULATE ASYMMETRIC CELL DIVISION IN MAIZE}

The development of stomatal complex in maize (Zea mays) is tightly associated with the coordination of asymmetric cell division. Stomatal complexes consist of a pair of guard cells flanked by a pair of subsidiary cells that regulate the function of guard cells. At first, guard mother cell (GMC), which eventually produces the guard cell pair, is formed by asymmetric cell division. Before formation of a guard cell pair, the nuclei of subsidiary mother cells (SMCs), which are lateral neighbors of GMC, are polarized toward GMC as well as forming a cortical F-actin patch at the boundaries between GMC and SMCs. And then the subsequent asymmetric cell division produces the subsidiary cells.

PANGLOSS1 (PAN1), a leucine-rich repeat receptor-like protein with inactive kinase domain, is localized in SMCs and newly formed subsidiary cells at sites of contact with GMCs. PAN1 and actin patches appeared after GMC formation and before nuclear polarization, and PAN1 patches were detectable before actin patches. Thus, PAN1 promotes the premitotic polarization of SMCs by receiving the cue from the GMC rather than intrinsic cues (Cartwright et al., 2009). Given the roles of ROPs in cell polarization and their association with RLKs, ROP signaling was expected to contribute to the polarization of SMCs. Nine ROPs were identified in maize. Two closely related ROPs, $\mathrm{ROP} 2$, and ROP9, play the roles in promoting the polarization of SMCs with PAN1. Although mutations in ROP2 or ROP9 alone caused no significant defects in the subsidiary cells, the double mutants rop2/rop2rop9/+ exhibited a slightly higher frequency of abnormal subsidiary cells. These mutations significantly enhanced the pan1 phenotype of SMC division. In SMCs, ROP proteins localize at sites of contact with GMC, which is similar to the PAN1 localization. ROP patches are formed after the formation of PAN1 patches. The accumulation of ROP in SMCs was altered in pan1 mutant, whereas the localization of PAN1 was not disturbed in rop2/rop2rop9/+ mutants. Furthermore, biochemical approaches revealed that PAN1 and ROPs are physically associated in maize leaf extracts (Humphries et al., 2011). These data suggest that ROP2 and ROP9 function downstream of PAN1 to promote the premitotic polarization of SMCs and the activity of these ROPs can be spatially regulated. PAN1 lacks a functional kinase domain, and thus is expected to act with other proteins in signal transduction, but currently there is no knowledge of signaling protein(s) that form a complex with PAN1. Upstream signaling molecules that regulate the PAN1-ROP2/ROP9 pathway are unknown. Recently, auxin signaling is shown to be involved in stomatal patterning in Arabidopsis (Le et al., 2014). Since the polar localization of maize PIN1 (ZmPIN1a) in subsidiary cells has been reported (Sutimantanapi et al., 2014), it is tempting to speculate that auxin might be an upstream molecule for PAN1ROP2/ROP9 pathway, similar to the TMK-ROP pathways in Arabidopsis.

\section{ROPs NEGATIVELY REGULATE ABSCISIC ACID SIGNALING}

The phytohormone ABA is a pivotal hormone that is involved in seed germination, plant development, and abiotic stress responses. Several putative ABA receptors including those localized to the PM have been proposed, and biochemical studies have also implied the existence of both cell surface and intracellular ABA receptors. However, only the pyrabactin resistancel (PYR)/PYR1-like (PYL)/regulatory component of ABA receptor (RCAR) family ABA receptors, which are localized to the cytoplasm or nucleus, have been widely accepted (Fujii et al., 2009; Ma et al., 2009; Nishimura et al., 2009; Park et al., 2009). The ABA-mediated interaction of these receptors with A-type protein phosphatase $2 \mathrm{Cs}$ (PP2Cs) results in the inhibition of PP2Cs, which are negative regulators of ABA signaling. The SNF1-related protein kinase 2 (SnRK2)-type protein kinases can then activate downstream signaling.

Evidence suggests that ROP GTPases also participate in the regulation of $A B A$ signaling mediated by the PYR/PYL/RCAR family ABA receptors. Expression of a dominant-positive mutant of ROP6/AtRac1 inhibited ABA-mediated stomatal closure and cytoskeletal reorganization, whereas expression of a DNform of ROP6 caused ABA response in an ABA-independent manner, indicating that ROP6 signaling negatively regulates ABA signaling (Lemichez et al., 2001). ROP10 loss of function mutants exhibit hypersensitivity to ABA in root elongation response, and stomatal closure. These mutants also displayed the enhanced seed dormancy, which is regulated by $\mathrm{ABA}$ and enhanced responses to ABA inhibition of seed germination. CA-ROP10 and DNROP10 mutants exhibited the insensitivity and hypersensitivity to ABA, respectively. The promoter-GUS analysis revealed that the ROP10 promoter activity in root tips is down-regulated by ABA (Zheng etal., 2002). These results suggest that a mutual inhibition between ROP10 signaling and ABA signaling. The ROP10-mediated pathway negatively regulates ABA signaling, while ROP10 transcripts are down-regulated by ABA. ROP11, which belongs to the same subfamily of ROP10, has been shown to be another negative regulator of $\mathrm{ABA}$ signaling in multiple ABA responses. The loss of function mutants of ROP11 exhibited hypersensitivity to ABA similarly to ROP10 mutants. Several lines of evidence show that ROP11 and CA-ROP11 directly interact with $\mathrm{ABI} 1$ and $\mathrm{ABI} 2$, which are members of the PP2Cs family (Li et al., 2012a,b; Yu et al., 2012). In vitro phosphatase activity assay showed that inhibition of ABI1 by RCAR1 was alleviated by CA-ROP11, and ROP11 enhanced the ABI2 activity (Li et al., 2012b; Yu etal., 2012). RopGEF1 and RopGEF4 have been reported to be upstream regulators of ROP11 in ABA-mediated stomatal closure ( $\mathrm{Li}$ and Liu, 2012). These data suggest that ROP signaling protects the hub of ABA signaling. 
Table 1 | A list of known and putative RLK-RopGEF-ROP combinations and their roles in various plant development.

\begin{tabular}{|c|c|c|c|c|c|c|}
\hline Signal & Receptor & RopGEF & ROP & Function & Plants & Reference \\
\hline Auxin & ABP1/TMK & Unknown & $\begin{array}{l}\text { ROP2, ROP4, } \\
\text { ROP6 }\end{array}$ & PC interdigitation & $\begin{array}{l}\text { Arabidopsis } \\
\text { thaliana }\end{array}$ & Xu et al. $(2010,2014)$ \\
\hline Auxin? & FER & RopGEF 1 & ROP2 & $\begin{array}{l}\text { Root hair } \\
\text { development }\end{array}$ & $\begin{array}{l}\text { Arabidopsis } \\
\text { thaliana }\end{array}$ & Duan etal. (2010) \\
\hline $\begin{array}{l}\text { LAT52?, } \\
\text { LeSTIG1, } \\
\text { LeSHY }\end{array}$ & $\begin{array}{l}\text { LePRK1/ } \\
\text { LePRK2 }\end{array}$ & LeKPP & Unknown & $\begin{array}{l}\text { Pollen tube } \\
\text { elongation }\end{array}$ & $\begin{array}{l}\text { Lycopersicon } \\
\text { esculentum }\end{array}$ & $\begin{array}{l}\text { Muschietti et al. (1994, 1998), Tang et al. (2002, } \\
\text { 2004), Kaothien et al. (2005) }\end{array}$ \\
\hline Unknown & AtPRK2a & $\begin{array}{l}\text { RopGEF } 1 \\
\text { RopGEF } 12\end{array}$ & ROP1 & $\begin{array}{l}\text { Pollen tube } \\
\text { elongation }\end{array}$ & $\begin{array}{l}\text { Arabidopsis } \\
\text { thaliana }\end{array}$ & $\begin{array}{l}\text { Gu et al. (2006), Zhang and McCormick (2007), } \\
\text { Chang etal. (2013) }\end{array}$ \\
\hline Unknown & PAN1 & Unknown & ROP2, ROP9 & $\begin{array}{l}\text { Stomatal complex } \\
\text { development }\end{array}$ & Zea mays & Cartwright etal. (2009), Humphries et al. (2011) \\
\hline Unknown & FER & RopGEF 1 & $\begin{array}{l}\text { ROP 10, } \\
\text { ROP } 11\end{array}$ & ABA signaling & $\begin{array}{l}\text { Arabidopsis } \\
\text { thaliana }\end{array}$ & $\begin{array}{l}\text { Zheng etal. (2002), Li and Liu (2012), Yu etal. } \\
\text { (2012), Li etal. (2012a,b) }\end{array}$ \\
\hline
\end{tabular}

Furthermore, mutations in FER caused hypersensitivity to ABA in root elongation responses and stomatal closure, as well as higher level of ROS accumulation in guard cells (Yu et al., 2012). Since RopGEF1 has been reported to interact with FER (Duan et al., 2010), FER-mediated signaling may regulate ABA responses through ROPs. Although ABA itself may be a regulator of ROP signaling in a negative feedback loop, the signals that activate specific ROPs in ABA signaling remain unknown. Given the occurrence of FER-RopGEF-ROP signaling at the PM, these results raise the possibility that ROPs may regulate $\mathrm{ABA}$ signaling at the PM too.

\section{CONCLUDING REMARKS}

Rho-like GTPase from plants signaling acts downstream of diverse signaling pathways such as auxin, ABA, and cytosolic calcium. Increasing evidence reveals that several ROPs are involved in RLK-mediated signaling. Given the diverse roles of ROP signaling in cellular processes such as actin accumulation, ordering of MTs, calcium concentration, $\mathrm{H}_{2} \mathrm{O}_{2}$ production, gene expression, all of which lead to symmetry breaking (Yang, 2002; Yang and Lavagi, 2012), multiple environmental and developmental signals that perceived by RLKs may be regulated by RLK-ROP modules. One of aspect yet to be examined remains the establishment of RLK-ROP modules as common features in plants. Thus far, FER, TMK, and AtPRK2a in Arabidopsis, LePRK1 and LePRK2 in tomato, and PAN1 in maize have been shown to be involved in ROP signaling, suggesting that the framework of RLK (or receptor like protein)-ROP is conserved among higher plants for acquisition of cell polarity (Table 1). The identification of RLKs or receptor-like proteins that interact with upstream regulators of ROPs like RopGEFs will be critical to further our understanding of ROP-mediated signaling. Another critical facet of RLK-ROP modules regards the roles of the interaction between RLKs and upstream regulators of ROP. Binding of the C terminal region of AtRopGEF12 with AtPRK2a results in the loss of C terminal inhibition of AtRopGEF12 activity and contributes to the activation of downstream ROP signaling in the tip growth of pollen tube (Zhang and McCormick, 2007; Chang et al., 2013). In root hair development, the inactive form of ROP2 preferentially binds to the complex of FER and AtRopGEF1, as compared with the active form of ROP2. The conversion of GDP-bound to GTP-bound ROP2 may be facilitated in an upstream signaldependent manner, and then the activated ROP2 may be released for downstream pathway (Duan et al., 2010). In PC interdigitation, auxin binding to ABP1 is shown to be required for the formation of the ABP1-TMK complex, as well as for the subsequent activation of ROP signaling (Xu et al., 2014). Understanding the complexity of RLK-ROP pathways will provide new insights into how a variety of signals can regulate ROP signaling.

\section{ACKNOWLEDGMENTS}

We thank Drs. Irene Lavagi, Shingo Nagawa, and members of Yang's laboratory for their valuable comments regarding the manuscript. This work is supported by grants from the U.S. National Institute of General Medical Sciences to Zhenbiao Yang (GM081451).

\section{REFERENCES}

Braun, N., Wyrzykowska, J., Muller, P., David, K., Couch, D., Perrot-Rechenmann, C., et al. (2008). Conditional repression of AUXIN BINDING PROTEIN1 reveals that it coordinates cell division and cell expansion during postembryonic shoot development in Arabidopsis and tobacco. Plant Cell 20, 2746-2762. doi: $10.1105 /$ tpc. 108.059048

Cartwright, H., Humphries, J., and Smith, L. (2009). PAN1: a receptor-like protein that promotes polarization of an asymmetric cell division in maize. Science 323, 649-651. doi: 10.1126/science.1161686

Chang, F., Gu, Y., Ma, H., and Yang, Z. (2013). AtPRK2 promotes ROP1 activation via RopGEFs in the control of polarized pollen tube growth. Mol. Plant 6, 1187-1201. doi: $10.1093 / \mathrm{mp} / \mathrm{sss} 103$

Chen, X., Naramoto, S., Robert, S., Tejos, R., Löfke, C., Lin, D., et al. (2012). ABP1 and ROP6 GTPase signaling regulate clathrin-mediated endocytosis in Arabidopsis roots. Curr. Biol. 22, 1326-1332. doi: 10.1016/j.cub.2012. 05.020 
Claussen, M., Lüthe, H., Blatt, M., and Böttger, M. (1997). Auxin-induced growth and its linkage to potassium channels. Planta 201, 227-234. doi: 10.1007/BF01007708

Craddock, C., Lavagi, I., and Yang, Z. (2012). New insights into Rho signaling from plant ROP/Rac GTPases. Trends Cell Biol. 22, 492-501. doi: 10.1016/j.tcb.2012.05.002

Dai, N., Wang, W., Patterson, S., and Bleecker, A. (2013). The TMK subfamily of receptor-like kinases in Arabidopsis display an essential role in growth and a reduced sensitivity to auxin. PLoS ONE 8:e60990. doi: 10.1371/journal. pone.0060990

Diekmann, W., Venis, M., and Robinson, D. (1995). Auxins induce clustering of the auxin-binding protein at the surface of maize coleoptile protoplasts. Proc. Natl. Acad. Sci. U.S.A. 92, 3425-3429. doi: 10.1073/pnas.92.8.3425

Duan, Q., Kita, D., Li, C., Cheung, A., and Wu, H. (2010). FERONIA receptor-like kinase regulates RHO GTPase signaling of root hair development. Proc. Natl Acad. Sci. U.S.A. 107, 17821-17826. doi: 10.1073/pnas.1005366107

Fu, Y., Gu, Y., Zheng, Z., Wasteneys, G., and Yang, Z. (2005). Arabidopsis interdigitating cell growth requires two antagonistic pathways with opposing action on cell morphogenesis. Cell 120, 687-700. doi: 10.1016/j.cell.2004.12.026

Fu, Y., Li, H., and Yang, Z. (2002). The ROP2 GTPase controls the formation of cortical fine F-actin and the early phase of directional cell expansion during Arabidopsis organogenesis. Plant Cell 14, 777-794. doi: 10.1105/tpc.001537

Fu, Y., Xu, T., Zhu, L., Wen, M., and Yang, Z. (2009). A ROP GTPase signaling pathway controls cortical microtubule ordering and cell expansion in Arabidopsis. Curr. Biol. 19, 1827-1832. doi: 10.1016/j.cub.2009.08.052

Fujii, H., Chinnusamy, V., Rodrigues, A., Rubio, S., Antoni, R., Park, S., et al. (2009). In vitro reconstitution of an abscisic acid signalling pathway. Nature 462, 660-664. doi: 10.1038/nature08599

Gu, Y., Fu, Y., Dowd, P., Li, S., Vernoud, V., Gilroy, S., et al. (2005). A Rho family GTPase controls actin dynamics and tip growth via two counteracting downstream pathways in pollen tubes. J. Cell Biol. 169, 127-138. doi: $10.1083 /$ jcb. 200409140

Gu, Y., Li, S., Lord, E., and Yang, Z. (2006). Members of a novel class of Arabidopsis Rho guanine nucleotide exchange factors control Rho GTPase-dependent polar growth. Plant Cell 18, 366-381. doi: 10.1105/tpc.105.036434

Gu, Y., Vernoud, V., Fu, Y., and Yang, Z. (2003). ROP GTPase regulation of pollen tube growth through the dynamics of tip-localized F-actin. J. Exp. Bot. 54, 93-101. doi: 10.1093/jxb/erg035

Gu, Y., Wang, Z., and Yang, Z. (2004). ROP/RAC GTPase: an old new master regulator for plant signaling. Curr. Opin. Plant Biol. 7, 527-536. doi: 10.1016/j.pbi.2004.07.006

Haruta, M., Sabat, G., Stecker, K., Minkoff, B., and Sussman, M. (2014). A peptide hormone and its receptor protein kinase regulate plant cell expansion. Science 343, 408-411. doi: 10.1126/science.1244454

Henderson, J., Bauly, J., Ashford, D., Oliver, S., Hawes, C., Lazarus, C., et al (1997). Retention of maize auxin-binding protein in the endoplasmic reticulum: quantifying escape and the role of auxin. Planta 202, 313-323. doi: $10.1007 / \mathrm{s} 004250050133$

Huang, W., Liu, H., McCormick, S., and Tang, W. (2014). Tomato pistil factor STIG1 promotes in vivo pollen tube growth by binding to phosphatidylinositol 3 -phosphate and the extracellular domain of the pollen receptor kinase LePRK2. Plant Cell 26, 2505-2523. doi: 10.1105/tpc.114.123281

Humphries, J., Vejlupkova, Z., Luo, A., Meeley, R., Sylvester, A., Fowler, J., etal. (2011). ROP GTPases act with the receptor-like protein PAN1 to polarize asymmetric cell division in maize. Plant Cell 23, 2273-2284. doi: 10.1105/tpc.111.085597

Hwang, J., Gu, Y., Lee, Y., and Yang, Z. (2005). Oscillatory ROP GTPase activation leads the oscillatory polarized growth of pollen tubes. Mol. Biol. Cell 16, 53855399. doi: 10.1091/mbc.E05-05-0409

Hwang, J., Vernoud, V., Szumlanski, A., Nielsen, E., and Yang, Z. (2008). A tip-localized RhoGAP controls cell polarity by globally inhibiting Rho GTPase at the cell apex. Curr. Biol. 18, 1907-1916. doi: 10.1016/j.cub.2008. 11.057

Jones, A., and Herman, E. (1993). KDEL-containing auxin-binding protein is secreted to the plasma membrane and cell wall. Plant Physiol. 101, 595-606.

Jones, M., Shen, J., Fu, Y., Li, H., Yang, Z., and Grierson, C. (2002). The Arabidopsis Rop2 GTPase is a positive regulator of both root hair initiation and tip growth. Plant Cell 14, 763-776. doi: 10.1105/tpc.010359
Kaothien, P., Ok, S., Shuai, B., Wengier, D., Cotter, R., Kelley, D., et al. (2005) Kinase partner protein interacts with the LePRK1 and LePRK2 receptor kinases and plays a role in polarized pollen tube growth. Plant J. 42, 492-503. doi: 10.1111/j.1365-313X.2005.02388.x

Le, J., Liu, X., Yang, K., Chen, X., Zou, J., Wang, H., et al. (2014). Auxin transport and activity regulate stomatal patterning and development. Nat. Commun. 5:3090. doi: $10.1038 /$ ncomms4090

Lee, Y., Szumlanski, A., Nielsen, E., and Yang, Z. (2008). Rho-GTPase-dependent filamentous actin dynamics coordinate vesicle targeting and exocytosis during tip growth. J. Cell Biol. 181, 1155-1168. doi: 10.1083/jcb.200801086

Lemichez, E., Wu, Y., Sanchez, J., Mettouchi, A., Mathur, J., and Chua, N. (2001). Inactivation of AtRacl by abscisic acid is essential for stomatal closure. Genes Dev. 15, 1808-1816. doi: 10.1101/gad.900401

Li, H., Lin, Y., Heath, R., Zhu, M., and Yang, Z. (1999). Control of pollen tube tip growth by a Rop GTPase-dependent pathway that leads to tip-localized calcium influx. Plant Cell 11, 1731-1742.

Li, H., Shen, J., Zheng, Z., Lin, Y., and Yang, Z. (2001). The Rop GTPase switch controls multiple developmental processes in Arabidopsis. Plant Physiol. 126, 670684. doi: 10.1104/pp.126.2.670

Li, Z., Kang, J., Sui, N., and Liu, D. (2012a). ROP11 GTPase is a negative regulator of multiple ABA responses in Arabidopsis. J. Integr. Plant Biol. 54, 169-179. doi: 10.1111/j.1744-7909.2012.01100.x

Li, Z., Li, Z., Gao, X., Chinnusamy, V., Bressan, R., Wang, Z., et al. (2012b). ROP11 GTPase negatively regulates ABA signaling by protecting ABI1 phosphatase activity from inhibition by the ABA receptor RCAR1/PYL9 in Arabidopsis. J. Integr. Plant Biol. 54, 180-188. doi: 10.1111/j.1744-7909.2012.01101.x

Li, Z., and Liu, D. (2012). ROPGEF1 and ROPGEF4 are functional regulators of ROP11 GTPase in ABA-mediated stomatal closure in Arabidopsis. FEBS Lett. 586, 1253-1258. doi: 10.1016/j.febslet.2012.03.040

Lin, D., Nagawa, S., Chen, J., Cao, L., Chen, X., Xu, T., et al. (2012). A ROP GTPase-dependent auxin signaling pathway regulates the subcellular distribution of PIN2 in Arabidopsis roots. Curr. Biol. 22, 1319-1325. doi: 10.1016/j.cub.2012. 05.019

Lin, Y., Wang, Y., Zhu, J., and Yang, Z. (1996). Localization of a Rho GTPase implies a role in tip growth and movement of the generative cell in pollen tubes. Plant Cell 8, 293-303. doi: 10.1105/tpc.8.2.293

Lin, Y., and Yang, Z. (1997). Inhibition of pollen tube elongation by microinjected Anti-Rop1Ps antibodies suggests a crucial role for Rho-type GTPases in the control of tip growth. Plant Cell 9, 1647-1659. doi: 10.1105/tpc.9.9.1647

Ma, Y., Szostkiewicz, I., Korte, A., Moes, D., Yang, Y., Christmann, A., et al. (2009). Regulators of PP2C phosphatase activity function as abscisic acid sensors. Science 324, 1064-1068. doi: 10.1126/science.1172408

Molendijk, A., Bischoff, F., Rajendrakumar, C., Friml, J., Braun, M., Gilroy, S., et al. (2001). Arabidopsis thaliana Rop GTPases are localized to tips of root hairs and control polar growth. EMBO J. 20, 2779-2788. doi: 10.1093/emboj/20. 11.2779

Muschietti, J., Dircks, L., Vancanneyt, G., and McCormick, S. (1994). LAT52 protein is essential for tomato pollen development: pollen expressing antisense LAT52 RNA hydrates and germinates abnormally and cannot achieve fertilization. Plant J. 6, 321-338. doi: 10.1046/j.1365-313X.1994.06030321.x

Muschietti, J., Eyal, Y., and McCormick, S. (1998). Pollen tube localization implies a role in pollen-pistil interactions for the tomato receptor-like protein kinases LePRK1 and LePRK2. Plant Cell 10, 319-330.

Nagawa, S., Xu, T., Lin, D., Dhonukshe, P., Zhang, X., Friml, J., et al. (2012). ROP GTPase-dependent actin microfilaments promote PIN1 polarization by localized inhibition of clathrin-dependent endocytosis. PLoS Biol. 10:e1001299. doi: 10.1371/journal.pbio.1001299

Napier, R., David, K., and Perrot-Rechenmann, C. (2002). A short history of auxinbinding proteins. Plant Mol. Biol. 49, 339-348. doi: 10.1023/A:1015259130955

Nibau, C., Wu, H., and Cheung, A. (2006). RAC/ROP GTPases: 'hubs' for signal integration and diversification in plants. Trends Plant Sci. 11, 309-315. doi: 10.1016/j.tplants.2006.04.003

Nishimura, N., Hitomi, K., Arvai, A., Rambo, R., Hitomi, C., Cutler, S., et al. (2009). Structural mechanism of abscisic acid binding and signaling by dimeric PYR1. Science 326, 1373-1379. doi: 10.1126/science.1181829

Park, S., Fung, P., Nishimura, N., Jensen, D., Fujii, H., Zhao, Y., et al. (2009). Abscisic acid inhibits type $2 \mathrm{C}$ protein phosphatases via the PYR/PYL family of START proteins. Science 324, 1068-1071. doi: 10.1126/science.1173041 
Philippar, K., Büchsenschütz, K., Edwards, D., Löffler, J., Lüthen, H., Kranz, E., et al. (2006). The auxin-induced $\mathrm{K}^{+}$channel gene $\mathrm{Zmk} 1$ in maize functions in coleoptile growth and is required for embryo development. Plant Mol. Biol. 61, 757-768. doi: 10.1007/s11103-006-0047-2

Pitts, R., Cernac, A., and Estelle, M. (1998). Auxin and ethylene promote root hair elongation in Arabidopsis. Plant J. 16, 553-560. doi: 10.1046/j.1365313x.1998.00321.x

Rahman, A., Hosokawa, S., Oono, Y., Amakawa, T., Goto, N., and Tsurumi, S. (2002). Auxin and ethylene response interactions during Arabidopsis root hair development dissected by auxin influx modulators. Plant Physiol. 130, 1908-1917. doi: 10.1104/pp.010546

Robert, S., Kleine-Vehn, J., Barbez, E., Sauer, M., Paciorek, T., Baster, P., et al. (2010). ABP1 mediates auxin inhibition of clathrin-dependent endocytosis in Arabidopsis. Cell 143, 111-121. doi: 10.1016/j.cell.2010.09.027

Shi, J., and Yang, Z. (2011). Is ABP1 an auxin receptor yet? Mol. Plant 4, 635-640. doi: $10.1093 / \mathrm{mp} / \mathrm{ssr} 050$

Shimomura, S. (2006). Identification of a glycosylphosphatidylinositol-anchored plasma membrane protein interacting with the C-terminus of auxin-binding protein 1: a photoaffinity crosslinking study. Plant Mol. Biol. 60, 663-677. doi: 10.1007/s11103-005-5471-1

Sutimantanapi, D., Pater, D., and Smith, L. (2014). Divergent roles for maize PAN1 and PAN2 receptor-like proteins in cytokinesis and cell morphogenesis. Plant Physiol. 164, 1905-1917. doi: 10.1104/pp.113.232660

Takahashi, K., Hayashi, K., and Kinoshita, T. (2012). Auxin activates the plasma membrane $\mathrm{H}^{+}$-ATPase by phosphorylation during hypocotyl elongation in Arabidopsis. Plant Physiol. 159, 632-641. doi: 10.1104/pp.112. 196428

Tang, W., Ezcurra, I., Muschietti, J., and McCormick, S. (2002). A cysteine-rich extracellular protein, LAT52, interacts with the extracellular domain of the pollen receptor kinase LePRK2. Plant Cell 14, 2277-2287. doi: 10.1105/tpc. 003103

Tang, W., Kelley, D., Ezcurra, I., Cotter, R., and McCormick, S. (2004). LeSTIG1, an extracellular binding partner for the pollen receptor kinases LePRK1 and LePRK2, promotes pollen tube growth in vitro. Plant J. 39, 343-353. doi: 10.1111/j.1365313X.2004.02139.x

Thiel, G., Blatt, M., Fricker, M., White, I., and Millner, P. (1993). Modulation of $\mathrm{K}^{+}$channels in Vicia stomatal guard cells by peptide homologs to the auxinbinding protein C terminus. Proc. Natl. Acad. Sci. U.S.A. 90, 11493-11497. doi: 10.1073/pnas.90.24.11493

Tromas, A., Braun, N., Muller, P., Khodus, T., Paponov, I., Palme, K., et al. (2009). The AUXIN BINDING PROTEIN 1 is required for differential auxin responses mediating root growth. PLoS ONE 4:e6648. doi: 10.1371/journal.pone. 0006648

Wengier, D., Valsecchi, I., Cabanas, M., Tang, W., McCormick, S., and Muschietti, J. (2003). The receptor kinases LePRK1 and LePRK2 associate in pollen and when expressed in yeast, but dissociate in the presence of style extract. Proc. Natl. Acad. Sci. U.S.A. 100, 6860-6865. doi: 10.1073/pnas.06317 28100

Xu, T., Dai, N., Chen, J., Nagawa, S., Cao, M., Li, H., et al. (2014). Cell surface ABP1-TMK auxin-sensing complex activates ROP GTPase signaling. Science 343, 1025-1028. doi: 10.1126/science. 1245125
Xu, T., Wen, M., Nagawa, S., Fu, Y., Chen, J., Wu, M., et al. (2010). Cell surface- and rho GTPase-based auxin signaling controls cellular interdigitation in Arabidopsis. Cell 143, 99-110. doi: 10.1016/j.cell.2010.09.003

Yan, A., Xu, G., and Yang, Z.-B. (2009). Calcium participates in feedback regulation of the oscillating ROP1 Rho GTPase in pollen tubes. Proc. Natl. Acad. Sci. U.S.A. 106, 22002-22007. doi: 10.1073/pnas.0910811106

Yang, Z. (2002). Small GTPases: versatile signaling switches in plants. Plant Cell 14(Suppl), S375-S388. doi: 10.1105/tpc.001065

Yang, Z. (2008). Cell polarity signaling in Arabidopsis. Annu. Rev. Cell Dev. Biol. 24, 551-575. doi: 10.1146/annurev.cellbio.23.090506.123233

Yang, Z., and Lavagi, I. (2012). Spatial control of plasma membrane domains: ROP GTPase-based symmetry breaking. Curr. Opin. Plant Biol. 15, 601-607. doi: 10.1016/j.pbi.2012.10.004

Yu, F., Qian, L., Nibau, C., Duan, Q., Kita, D., Levasseur, K., et al. (2012). FERONIA receptor kinase pathway suppresses abscisic acid signaling in Arabidopsis by activating ABI2 phosphatase. Proc. Natl. Acad. Sci. U.S.A. 109, 14693-14698. doi: 10.1073/pnas.1212547109

Zhang, D., Wengier, D., Shuai, B., Gui, C., Muschietti, J., McCormick, S., et al. (2008). The pollen receptor kinase LePRK2 mediates growth-promoting signals and positively regulates pollen germination and tube growth. Plant Physiol. 148, 1368-1379. doi: 10.1104/pp.108.124420

Zhang, Y., and McCormick, S. (2007). A distinct mechanism regulating a pollenspecific guanine nucleotide exchange factor for the small GTPase Rop in Arabidopsis thaliana. Proc. Natl. Acad. Sci. U.S.A. 104, 18830-18835. doi: 10.1073/pnas.0705874104

Zhao, X., Wang, Q., Li, S., Ge, F., Zhou, L., McCormick, S., et al. (2013). The juxtamembrane and carboxy-terminal domains of Arabidopsis PRK2 are critical for ROP-induced growth in pollen tubes. J. Exp. Bot. 64, 5599-5610. doi: 10.1093/jxb/ert323

Zheng, Z., Nafisi, M., Tam, A., Li, H., Crowell, D., Chary, S., et al. (2002). Plasma membrane-associated ROP10 small GTPase is a specific negative regulator of abscisic acid responses in Arabidopsis. Plant Cell 14, 2787-2797. doi: $10.1105 /$ tpc. 005611

Conflict of Interest Statement: The authors declare that the research was conducted in the absence of any commercial or financial relationships that could be construed as a potential conflict of interest.

Received: 10 July 2014; paper pending published: 31 July 2014; accepted: 20 August 2014; published online: 22 September 2014.

Citation: Miyawaki KN and Yang Z (2014) Extracellular signals and receptorlike kinases regulating ROP GTPases in plants. Front. Plant Sci. 5:449. doi: 10.3389/fpls.2014.00449

This article was submitted to Plant Traffic and Transport, a section of the journal Frontiers in Plant Science.

Copyright (c) 2014 Miyawaki and Yang. This is an open-access article distributed under the terms of the Creative Commons Attribution License (CC BY). The use, distribution or reproduction in other forums is permitted, provided the original author(s) or licensor are credited and that the original publication in this journal is cited, in accordance with accepted academic practice. No use, distribution or reproduction is permitted which does not comply with these terms. 